\title{
Design of the GIF Antenna Based on Phased-Array Technology
}

\author{
Yang Jia, Hong-bin Ren ${ }^{b}$ and Jing-xin Liu ${ }^{c}$ \\ Air and Missile Defense College, Air Force Engineering University, Shanxi Xi'an, 710051, China \\ ajiyangjs@163.com, ${ }^{b} 18706766533 @ 163 . c o m,{ }^{c}$ puma330@126.com
}

Keywords: Fuze, Seeker, Integrated design, Phased-array antenna.

\begin{abstract}
The need of antenna design and the basic principle of phased-array antenna are analysised. Then the rectangular-grid-plane phased array with circular boundary design of antenna which is shared by seeker and fuze is put forward. By choosing the parameters and number of elements, the antenna adaptively changes the beam pointing and flap width to meet two different working requirements of the seeker and fuze. Finally, the performance of Guidance Integrated Fuzing (GIF) antenna is simulated and validated. The results indicate that the antenna adapts to the needs of seeker and fuze, and it is a new sort to improve the coordination efficiency of fuze and warhead.
\end{abstract}

\section{Introduction}

With the continuous developments of anti-aircraft missiles and high maneuvering targets, missile-target encountering environment is becoming more and more complex. Effective measures must be taken to improve the coordinated efficiency of the fuze and the warhead ${ }^{[1-3]}$.

The Guidance Integrated Fuzing (GIF) can be interpreted that the fuze and the seeker are considered and designed comprehensively in many ways such as working principle, structure design, circuit design, signal processing, etc. Integrated design of fuze and seeker antenna can make sure that fuze makes full use of the related information to adaptively control the detonation of warhead and make the fragment aim at the target. The seeker can not only guide missile flight but also control the detonation of warhead adaptively. The central content of the GIF is that the fuze and the warhead share the information and equipment with each other. This technology is presented to improve the coordinated efficiency of the fuze and the warhead and make sure anti-interference performance and reliability of the detonating system.

The most important technology of the GIF on hardware is the integration design of the target detection device. It should not only ensure that the fuze can obtain the information accurately but also ensure that the seeker can search, capture and track the target. The beam inclination angle and the beam width are two important parameters during the design of antenna.

\section{Integrated Design of Antenna}

The theory of the integrated design of fuze and seeker antenna based on phased-array technology is shown as Fig.1. The system is based on the active phased-array antenna and each element is made up of the transceiver, the phase shifter and power dispatcher, etc. The power distribution and composition system presents transmit power and forms receiving beam. Beam controller is the particular component of phased-array seeker and fuze. It substitutes for machine scanning system. Under the control of the information processing machine, beam controller calculates controlling code which each phase shifter of antenna element needs and transmits it to the driver.

In the diagram, the fuze and seeker share the phased-array antenna, signal and data processing equipment. At the beginning of the approach of missile and target, antenna system chooses working condition of the seeker to provide information for the system of guidance and control for missile. At the stage of the terminal guidance, antenna system switches to the working condition of fuze. Make sure the fuze detect the target and control adaptively the detonation of warhead. 


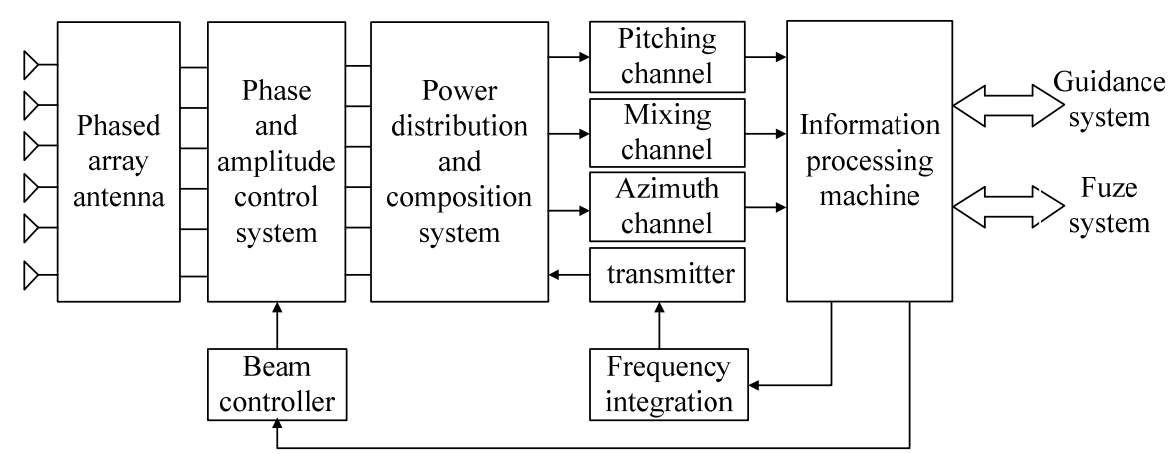

Fig. 1 The function block diagram of the GIF antenna

\section{Theory of Phased-array Antenna}

\subsection{Basal principle ${ }^{[4-7]}$}

Phased-array antenna can scan simultaneously in two directions which are pitch plane and bearing plane. The chart of rectangle phased-array antenna on plane and 3D beam-scanning picture are shown as Fig.2. On xoy plane, the sum of antenna array elements is $M \times N$ and the spacing between the elements can be described with ${ }^{d_{x}}$ and $d_{y}$
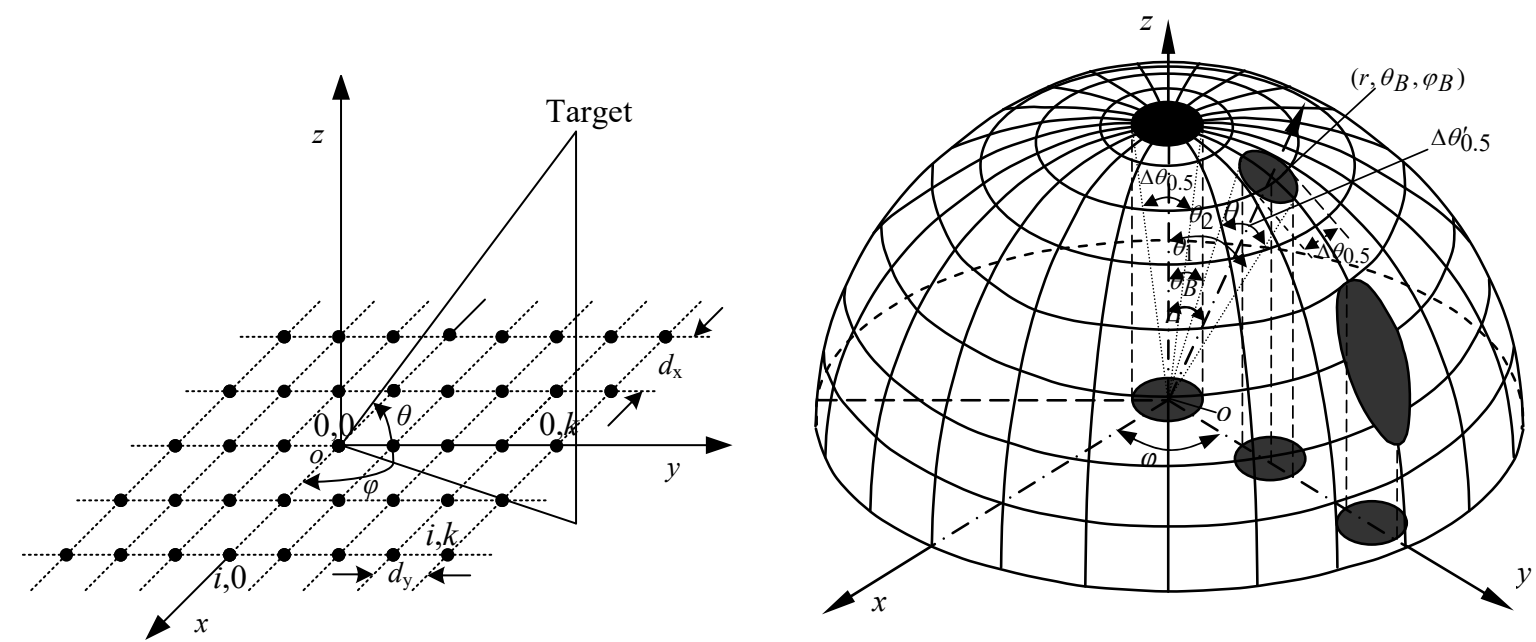

Fig.2 Rectangle phased-array antenna on plane and 3D beam-scanning picture

Supposing the position coordinate of target is $(\cos \theta \cos \varphi, \cos \theta \sin \varphi, \sin \theta)$, the pattern of the phased-array antenna $F\left(\alpha_{x}, \alpha_{y}\right)$ can be defined as follows:

$$
F\left(\alpha_{x}, \alpha_{y}\right)=\sum_{i} \sum_{k} \alpha_{i k} e^{j\left[i\left(\frac{2 \pi}{\lambda} d_{x} \cos \theta \cos \varphi-\Delta \phi_{B \beta}\right)+k\left(\frac{2 \pi}{\lambda} d_{y} \cos \theta \sin \varphi-\Delta \phi_{B \alpha}\right)\right]}
$$

Where $\Delta \phi_{B \beta}$ is the phase difference of two adjacent elements in the $\mathrm{X}$ axis direction and $\Delta \phi_{B \alpha}$ is the phase in the $\mathrm{Y}$ axis direction. If $(2 \pi / \lambda) d_{x} \cos \theta \cos \varphi=\Delta \phi_{B \beta}$ and $(2 \pi / \lambda) d_{y} \cos \theta \sin \varphi=\Delta \phi_{B \alpha}$, the maximum value of the pattern of the phased-array antenna can be derived. Therefore, changing $\Delta \phi_{B \beta}$ and $\Delta \phi_{B \alpha}$ can lead to the adjustment of beam direction and beam scanning.

\subsection{Beam width}

Reference to Fig.2, the beam width in $\varphi$ direction remains unchanged even if scanning angle changes. Yet the beam width in $\theta$ direction will broaden if the scanning angle $\theta_{B}$ increases.

When phased-array antenna doesn't scan, its beam will be like a pencil and half power beam width is $\Delta \theta_{0.5}$. The radius of projected circle on a plane can be expressed as $r \sin \left(\Delta \theta_{0.5} / 2\right)$. When the direction of beam is $\left(\theta_{B}, \varphi_{B}\right)$, beam only make the operation of translation on xoy plane according to the geometrical theory, the beam width can get through geometrical operation as follows:

$$
\left\{\begin{array}{l}
\sin \theta_{1}=\sin \theta_{B}-\sin \left(\Delta \theta_{0.5} / 2\right) \\
\sin \theta_{2}=\sin \theta_{B}+\sin \left(\Delta \theta_{0.5} / 2\right)
\end{array}\right.
$$


Based on the above operation, the beam width $\Delta \theta_{0.5}^{\prime}$ can be obtained as follows:

$$
\Delta \theta_{0.5}^{\prime}=\theta_{2}-\theta_{1}=\arcsin \left[\sin \theta_{B}+\sin \left(\Delta \theta_{0.5} / 2\right)\right]-\arcsin \left[\sin \theta_{B}-\sin \left(\Delta \theta_{0.5} / 2\right)\right]
$$

\subsection{Spacing between array elements}

Reference to the rectangular grids, it is necessary to choose wide spacing as far as possible to reduce the number of elements needed if there is not grating lobe. If working frequency and maximum scanning angle $\theta_{m}$ are confirmed, the maximum spacing of antenna elements $d_{x}, d_{y}$ can be obtained as follows:

$$
d_{x}, d_{y} \leq \lambda /\left(1+\left|\sin \theta_{m}\right|\right)
$$

\subsection{Taylor linear source integrated model}

Reference to the linear source, ideal space factor ${ }^{[8-9]}$ can be replaced with Taylor space factor.

$$
F(m, A, \bar{n})= \begin{cases}\frac{[(\bar{n}-1) !]^{2}}{(\bar{n}-1+m) !(\bar{n}-1-m) !} \prod_{n=1}^{\bar{n}-1}\left(1-\frac{m^{2}}{\sigma^{2}\left[A^{2}+(n-1 / 2)^{2}\right.}\right) & |m|<\bar{n} \\ 0 & |m| \geq \bar{n}\end{cases}
$$

Where $\sigma$ is broadening factor and it can be defined as follows:

$$
\sigma=\bar{n} /\left(\sqrt{A^{2}+\left(\bar{n}-\frac{1}{2}\right)^{2}}\right)
$$

$\bar{n}$ is the divided point between the area of uniform side lobes and the area of various side lobes. It should be ensured that the increase of $\bar{n}$ will lead to the decrease of $\sigma$. So the requirement of $\bar{n}$ is provided as follows: $\bar{n} \geq 2 A^{2}+1 / 2$.

Therefore incentive amplitude of Taylor array elements can be expressed as:

$$
f\left(p_{n}\right)=1+2 \sum_{m=1}^{\bar{n}-1} F(m, A, \bar{n}) \cos \left(m p_{n}\right)
$$

with $\quad p_{n}=\left\{\begin{array}{lll}2 \pi d n / L & n=0,1, \cdots, N & N \text { is an odd number } \\ 2 \pi d(2 n+1) / L & n=0,1, \cdots, N-1 & N \text { is an even number }\end{array}\right.$

The main lobe width of Taylor array pattern can be derived as:

$$
\Delta \theta_{0.5}=2 \arcsin \left(\frac{\sigma \lambda}{\pi L}\left[(\operatorname{arccosh} R)^{2}-(\operatorname{arccosh}(R / \sqrt{2}))^{2}\right]^{\frac{1}{2}}\right)
$$

Supposing aperture is based on the Taylor distribution, the maximum distance between elements without grating lobes is given by:

$$
d<\zeta \frac{\lambda}{1+\left|\sin \theta_{\max }\right|}
$$

Where $\zeta$ is reduced factor of array spacing and it can be defined as follows: $\zeta=\left(N-\sqrt{1+\xi^{2}}\right) / N$ and $\xi$ is zero adjustment parameter of the pattern and it can be obt ained by solving the following equation.

$$
\sinh (\pi \xi)=\pi \xi 10^{(R-13.2614) / 20}
$$

\section{Installed Design of Antenna}

The GIF antenna is required not only to achieve the aim of guidance and control, but also to finish detonating fuze. From the perspective of structure and installed ways of integrated antenna, rectangular-grid-plane antenna which has circular boundary can be adopted and the way of installing is shown as Fig.3.

The design method of antenna was developed by separating the caliber. First, two main planes ( $\varphi=0^{\circ}$ and $\varphi=90^{\circ}$ ) can be designed according to the Taylor linear source integrated model and corresponding currents are obtained. If the two kinds of currents are multiplied together, the current distribution of each element will be obtained and each technique index can achieve or even precede the prescribed standard. 
Technical parameter of the GIF antenna is shown as follows: working frequency $f=35 \mathrm{GHz}$; the range of beam width $\Delta \theta_{0.5}^{\prime}$ is from $3^{\circ}$ to $8^{\circ}$; the range of scanning angle in $\theta$ direction is $\pm 65^{\circ}$ and in $\varphi$ direction is $360^{\circ}$; antenna gain is greater than $28 \mathrm{~dB}$; Side lobe voltage is less than $-30 \mathrm{~dB}$.

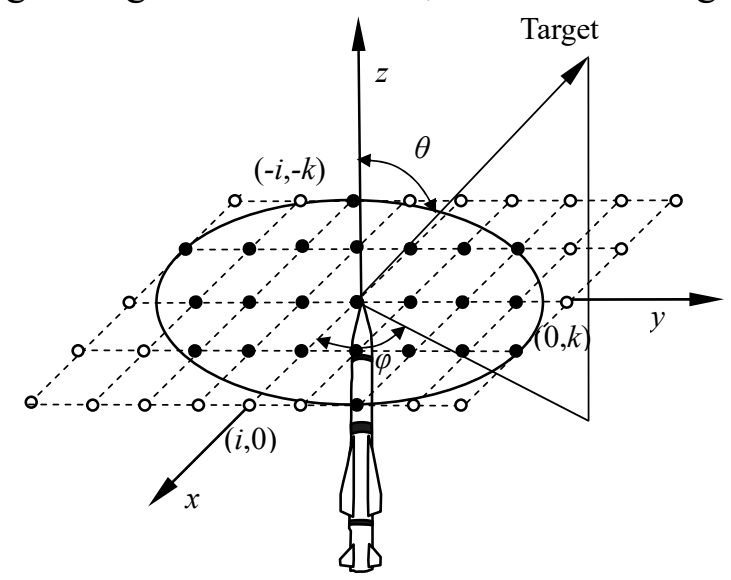

Fig.3 sketch map of antenna setting

According to the above technical requirements and installation conditions, square array antenna is designed firstly and the distribution of antenna caliber field is designed based on Taylor array. Linear source is designed according to the following steps.

(1) To choose relative parameters of Taylor array. When $\bar{n}=5, R=50$, side lobe voltage can be expressed as follows:

$$
\begin{aligned}
& E_{p}=-10 \lg R^{2}=-33.9794 \mathrm{~dB}<-30 \mathrm{~dB} \\
& A=\operatorname{arccosh} R / \pi=1.4658
\end{aligned}
$$

Thereupon

$$
\sigma=\bar{n} / \sqrt{A^{2}+(\bar{n}-0.5)^{2}}=1.0565
$$

(2) Considering the conditions of the beam width, gain and grating lobe, we can quantify caliber size and the distance between elements. When $L=18 \mathrm{~cm}$ and $N=19, d / \lambda$ can be obtained, that is 0.4912. The beam width $\Delta \theta_{0.5}$ of the antenna without scanning can get through the operation as follows:

$$
\Delta \theta_{0.5}=2 \arcsin \left(\frac{\sigma \lambda}{\pi L}\left[(\operatorname{arccosh} R)^{2}-(\operatorname{arccosh}(R / \sqrt{2}))^{2}\right]^{\frac{1}{2}}\right)=3.2171^{\circ}
$$

When the antenna is scanning, $\Delta \theta_{0.5 \max }^{\prime}$ can be obtained according to the Eq.(3).

$\Delta \theta_{0.5}^{\prime}=\arcsin \left[\sin 65^{\circ}+\sin \left(\Delta \theta_{0.5} / 2\right)\right]-\arcsin \left[\sin 65^{\circ}-\sin \left(\Delta \theta_{0.5} / 2\right)\right]=7.6675^{\circ}<8^{\circ}$

Directivity factor of phased-array antenna is connected with scanning angle and minimum $D_{\min }$ can be obtained as follows:

$$
D_{\min }=\pi D_{1}^{2} \cos \theta_{B \max }=2342.0529
$$

Where $D_{1}$ is directivity factor of linear source. Considering that the aperture of the antenna is small, the efficiency can not be very high. So supposing the efficiency $\eta=0.6$, the gain can be obtained get through the following operation.

$G_{\min }=10 \lg \left(D_{\min } \cdot \eta\right)=31.4775 \mathrm{~dB}>28 \mathrm{~dB}$

According to Eq.(11), we can easily obtain

$$
\zeta=\left(N-\sqrt{1+\xi^{2}}\right) / N=0.9467
$$

Then we have

$$
\frac{d}{\lambda}=0.4912<\zeta \frac{1}{1+\left|\sin \theta_{\max }\right|}=0.5119
$$

From Eq.(20), we can see that the antenna will not cause grating lobes.

(3) To calculate incentive amplitude of Taylor array elements.

Considering the symmetry of Taylor distribution, we can choose the $L / 2$ position as the position 
of reference point and only need to calculate half incentive amplitude. Performing integration by part for Eq.(8), and taking the $\sigma$ and $A$ calculated into consideration, we have

$$
F(1, A, 5)=0.334343 \quad F(2, A, 5)=-0.015765 \quad F(3, A, 5)=0.003948 \quad F(4, A, 5)=-0.000501
$$

Substituting the above calculation into Eq.(10), the tabulation of incentive amplitude of Taylor array elements is shown as Tab.1.

Then the specific design of square linear source array is completed. The diameter of plane is $18 \mathrm{~cm}$ and the number of elements is $45 \times 45$. The distribution of antenna aperture field is shown as Fig.4. If the GIF antenna system chooses working mode of seeker, all elements will be operated. When the system switches to the working condition of fuze, the number of elements will decrease and there will be only 21 elements to run to ensure the fuze deal with the information quickly.

Table 1 Incentive amplitude of Taylor array elements

\begin{tabular}{|c|c|c|c|c|c|c|c|c|}
\hline elements & $f\left(p_{0}\right)$ & $f\left(p_{1}\right)$ & $f\left(p_{2}\right)$ & $f\left(p_{3}\right)$ & $f\left(p_{4}\right)$ & $f\left(p_{5}\right)$ & $f\left(p_{6}\right)$ & $f\left(p_{7}\right)$ \\
\hline amplitude & 1.644051 & 1.638234 & 1.620888 & 1.592322 & 1.553047 & 1.503761 & 1.445325 & 1.378732 \\
\hline elements & $f\left(p_{8}\right)$ & $f\left(p_{9}\right)$ & $f\left(p_{10}\right)$ & $f\left(p_{11}\right)$ & $f\left(p_{12}\right)$ & $f\left(p_{13}\right)$ & $f\left(p_{14}\right)$ & $f\left(p_{15}\right)$ \\
\hline amplitude & 1.305063 & 1.225446 & 1.141029 & 1.052973 & 0.962470 & 0.870794 & 0.779366 & 0.689820 \\
\hline elements & $f\left(p_{16}\right)$ & $f\left(p_{17}\right)$ & $f\left(p_{18}\right)$ & $f\left(p_{19}\right)$ & $f\left(p_{20}\right)$ & $f\left(p_{21}\right)$ & $f\left(p_{22}\right)$ & \\
\hline amplitude & 0.604042 & 0.524170 & 0.452529 & 0.391504 & 0.343365 & 0.310063 & 0.293033 & \\
\hline
\end{tabular}
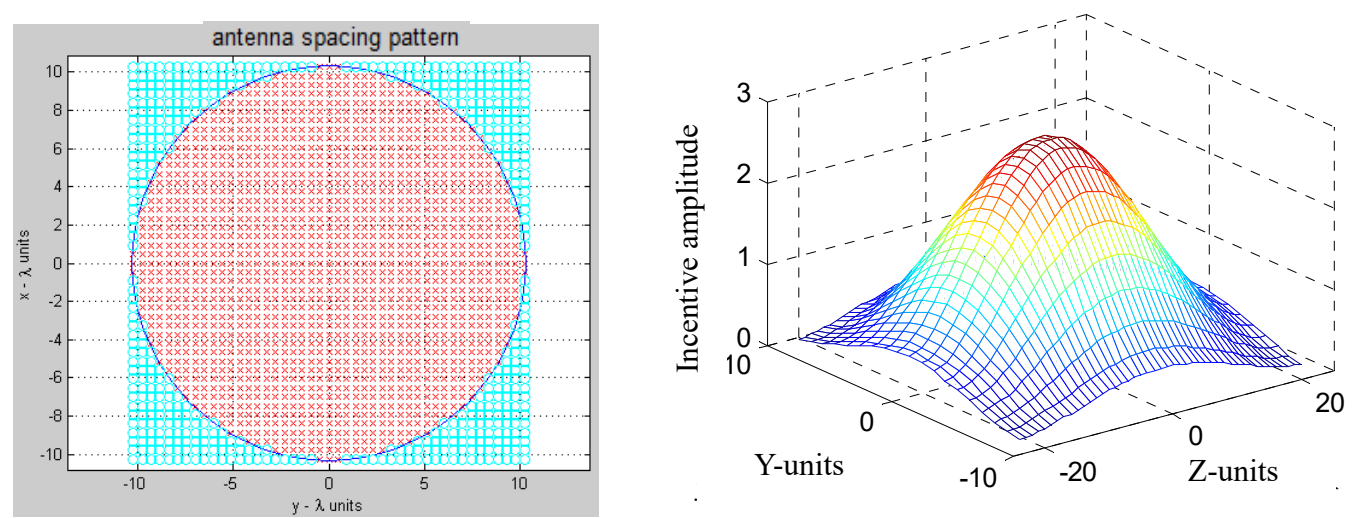

Fig.4 Installation diagram of antenna element and distribution of antenna aperture field

\section{Experiments and Simulation}

To describe scanning situation of the GIF antenna system preferably, we can suppose $u=\sin \theta \cos \varphi, v=\sin \theta \sin \varphi$ that is $\varphi=a \tan (u / v), \theta=a \sin \sqrt{u^{2}+v^{2}}$. The figure of converting the spherical coordinates to $\mathrm{U}, \mathrm{V}$ space is shown as Fig.5. Therefore $\theta, \varphi$ can be replaced with $u, v$ and scanning capability of beam will be expressed intuitively. Fig.6 is antenna gain direction of 3D graphs and line graphs at seeker state while beam-scanning angle $\theta_{B}=\varphi_{B}=0^{\circ}, \theta_{B}=65^{\circ} \varphi_{B}=0^{\circ}$

and $\theta_{B}=45^{\circ} \varphi_{B}=30^{\circ}$ based on size $\mathrm{N}_{\mathrm{r}}=45, d_{x}=d_{y}=0.4667 \lambda$ that is the working mode of seeker. Fig7 is antenna gain direction of $3 \mathrm{D}$ graphs and line graphs at seeker state while beam-scanning angle $\theta_{B}=\varphi_{B}=0^{\circ}, \theta_{B}=65^{\circ} \varphi_{B}=0^{\circ}$ and $\theta_{B}=45^{\circ} \varphi_{B}=30^{\circ}$ based on size $\mathrm{N}_{\mathrm{r}}=21, d_{x}=d_{y}=0.4667 \lambda$ that is the working mode of fuze.

In these figures, the beam shapes based on several different angles are presented. Line graphs describe the value of antenna gain in different conditions. The GIF antenna will form the beams with different shapes and direction based on different azimuth angles and elevation angles. Moreover, it can scan in two working modes that are seeker and fuze. 


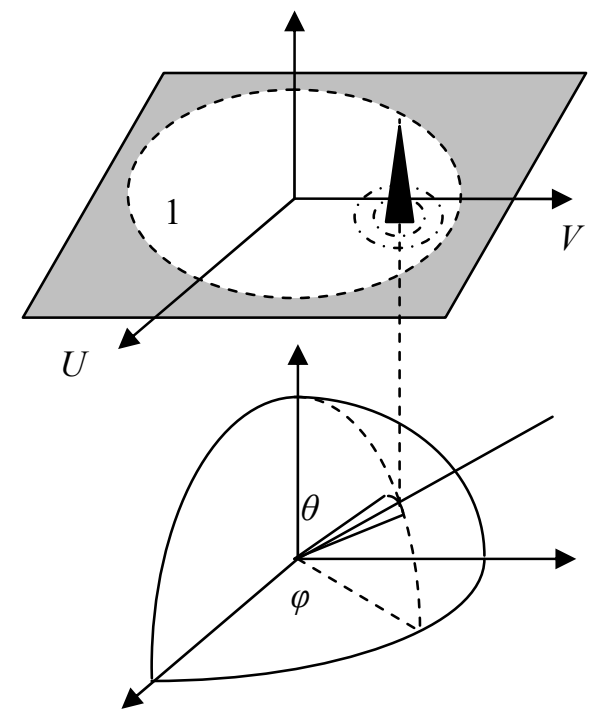

Fig.5 Converting the spherical coordinates to U,V space
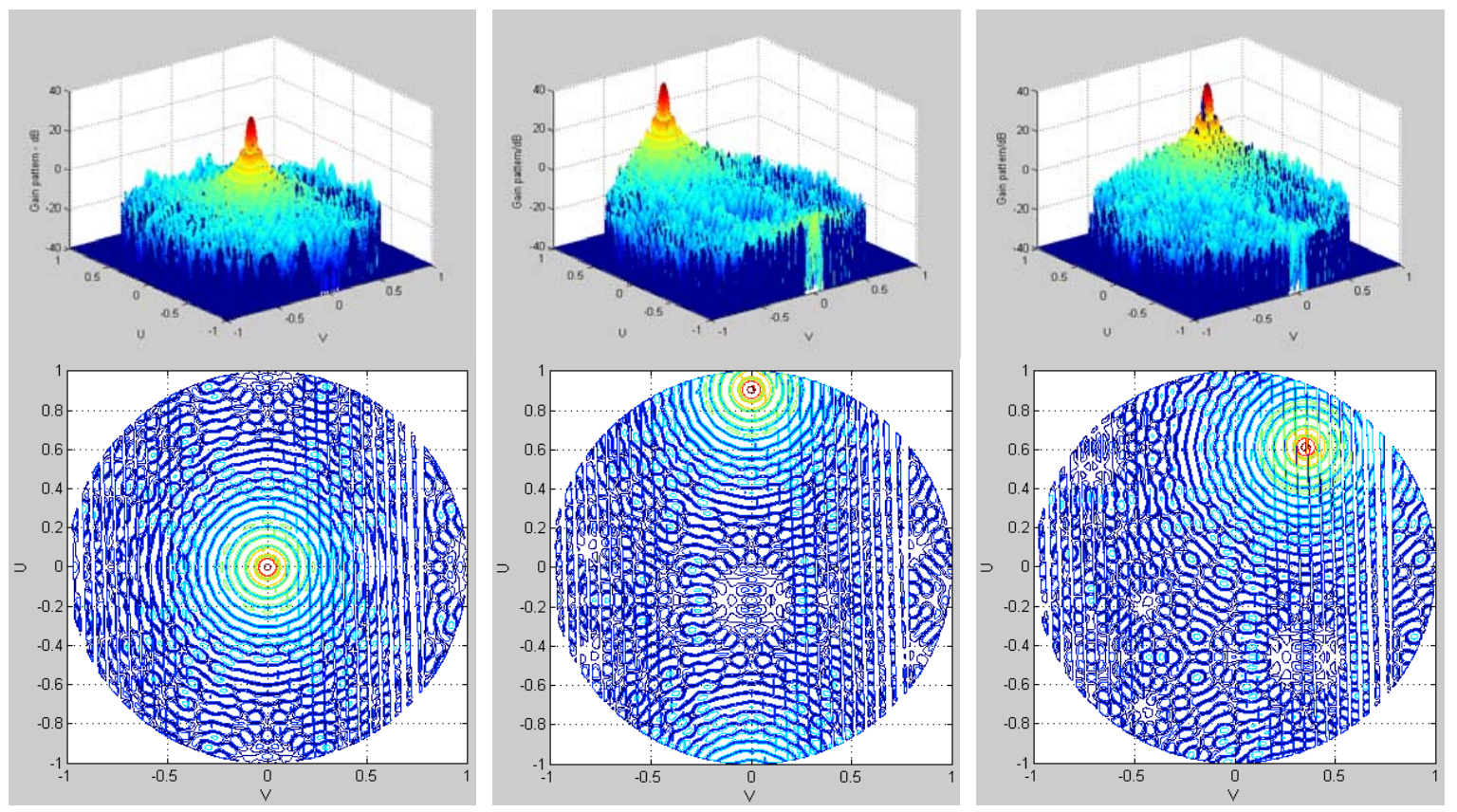

Fig.6 antenna gain directions of 3D graphs and line graphs at seeker state while beam-scanning angle $\theta_{B}=\varphi_{B}=0^{\circ}, \theta_{B}=65^{\circ}, \varphi_{B}=0^{\circ}$ and $\theta_{B}=45^{\circ}, \varphi_{B}=30^{\circ}$
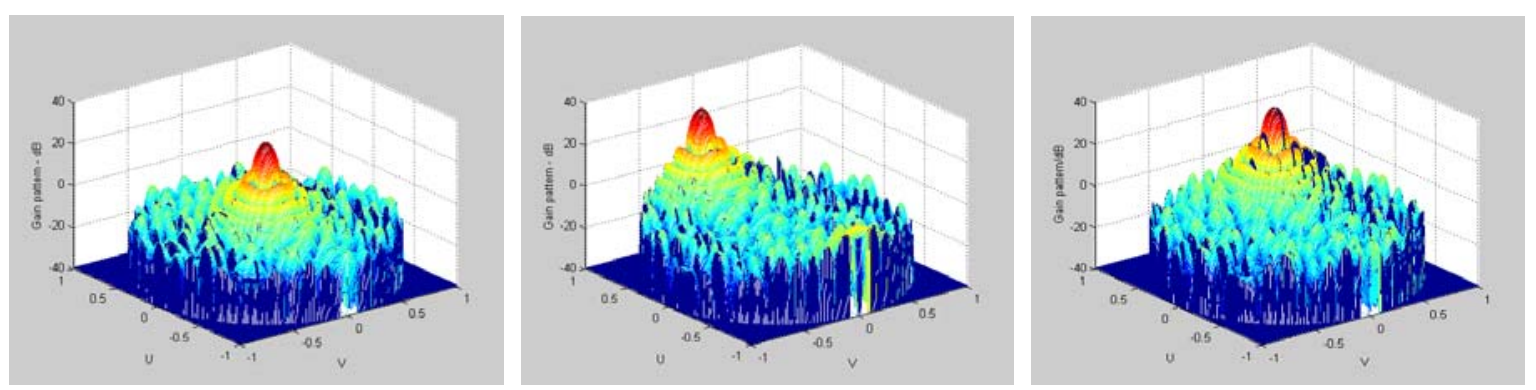

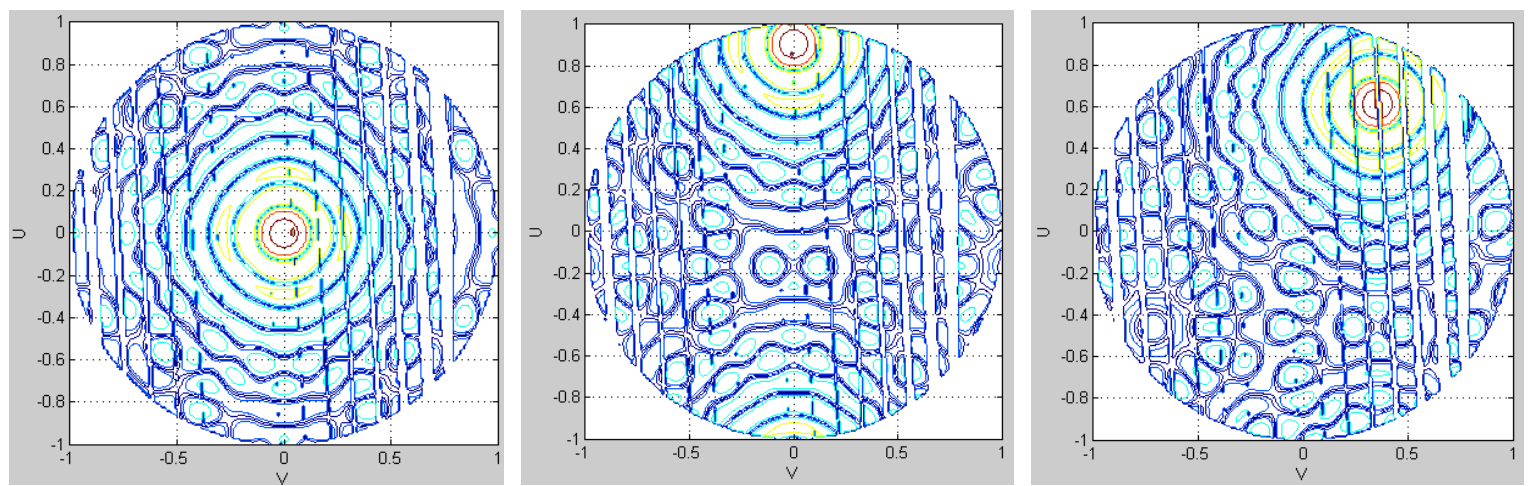

Fig.7 antenna gain directions of 3D graphs and line graphs at fuze state while beam-scanning angle $\theta_{B}=\varphi_{B}=0^{\circ} \theta_{B}=65^{\circ}, \varphi_{B}=0^{\circ}$ and $\theta_{B}=45^{\circ}, \varphi_{B}=30^{\circ}$

\section{Summary}

Based on the features of Phased-array antenna, we design the GIF antenna system that can not only adapt to active-radar seeker but also meet the technical requirements of fuze. At the beginning of the approach of missile and target, antenna system chooses working condition of the seeker to provide information for the system of guidance and control for missile. At the stage of the terminal guidance, antenna system switches to the working condition of fuze. Make sure the fuze detect the target and control adaptively the detonation of warhead. The information of the target can be made comprehensive use of and volume and weight of will decrease. The coordinated efficiency of fuze and warhead can be improved very well. However this technology will cause some problems such as it is very difficult to choose appropriate time to switch different working modes. Moreover phased-array antenna is very expensive and miniaturization is very difficult, which is needed to be solved step by step in the future.

\section{Reference:}

[1] ZHU Xue-ping, MENG Jiang-hao, XU Tao, YANG Jun. Integration Guidance Technology of Phased Array Radar Seeker Based on Maneuvering Target Tracking [J]. College of Astronautics Northwestern Polytechnical University, 2013.5:695-699.

[2] FAN Hui-tao, YAN Jun. Development and outlook of active electronically scanned array guidance technology [J]. Acta Aeronautica Et Astronautica Sinica, 2015.9:2807-2813.

[3] Fan Hui-tao. Design principle of air to air missile [M]. Beijing: Aviation Industry Press, 2013(in Chinese)

[4] Mark Engel, Eith Lewis, HowieWendt. GIF Guidance Integrated Fuze. NDIA 47th Fuze Conference

[5] LIU Hua-ting, GUO Yan-chang, LIU Zhen-guo. Reduction of spur in DDS based on appropriate random phasing in phased arrays [J]. Journal of Microwaves, 2014.30(4):72-74.

[6] Qiu Yuan-Yuan, Xu Yang. Optimization of side-lobes generated from a phased array using Gaussian superposition technique [J]. Journal of Nanjing University(Natural Sciences). 2013.6:782-787

[7] FU Hao, LI Xiao-hao, LI hui-lian. Research on the technologies of conformal phased array antenna [J]. Popular Science \& Technology, 2014.8:23-26.

[8] WANG Xuan, DENG Jia-hao, LI Hui, LU Man-jun. Terminal Target Contour Reconstruction Algorithm Based on RF Imaging for GIF [J]. Acta Armamentarii, 2015.9:1624-1631.

[9] DENG Fang-yi, ZOU Yi. Simulation of stable tracking control for gimbal of phase array seeker [J]. Systems Engineering and Electronics, 2013,2:402-407. 
[10]Aleksey K, Sergey K, Sergey S. A new technique for sandwich antenna radomes analysis[C]//2014 Loughborough Antennas and Propagation Conference. 2014:325-328. 